\title{
Réception de La Lanterne par la presse canadienne-française
}

\section{Francis Parmentier}

Volume 34, numéro 2, septembre 1980

URI : https://id.erudit.org/iderudit/303860ar

DOI : https://doi.org/10.7202/303860ar

Aller au sommaire du numéro

\section{Éditeur(s)}

Institut d'histoire de l'Amérique française

\section{ISSN}

0035-2357 (imprimé)

1492-1383 (numérique)

Découvrir la revue

\section{Citer cette note}

Parmentier, F. (1980). Réception de La Lanterne par la presse

canadienne-française. Revue d'histoire de l'Amérique française, 34(2), 269-274.

https://doi.org/10.7202/303860ar d'utilisation que vous pouvez consulter en ligne.

https://apropos.erudit.org/fr/usagers/politique-dutilisation/ 
II

\title{
RÉCEPTION DE LA LANTERNE PAR LA PRESSE CANADIENNE-FRANÇAISE
}

\author{
FRANCIS PARMENTIER \\ Département de français \\ Université du Québec à Trois-Rivières
}

La Lanterne canadienne se voulait l'ennemie de la bêtise et de l'hypocrisie mais, contrairement à son illustre homonyme parisien [la Lanterne de Rochefort, bête noire du Second Empire] dont la renommée avait traversé l'Atlantique, la Lanterne ne trouva que de faibles échos à l'extérieur du Canada et sa vie fut de courte durée. Toutefois, sans déchaîner les polémiques, elle piqua la presse catholique et réactionnaire du Canada français au point d'entraîner certaines répliques frisant la semonce. C'est l'analyse du contenu de ces répliques qui constituera l'essentiel de notre propos.

$\mathrm{Au}$ lieu de dépouiller toute la presse canadienne des années 1868-1869, nous avons porté notre attention sur les journaux suivants: Le Pays, Le Nouveau-Monde, Le Courrier du Canada, Le Journal des Trois-Rivières, L'Ordre, Le Canadien, L'Événement, Le Patriote et La Minerve, auxquels Buies fait référence dans la Lanterne.

Notons que les journaux énumérés ci-dessus ont, dans l'ensemble, peu réagi à la Lanterne. À part quelques entrefilets ironiques, les confrères de Buies restèrent muets sur cette singulière prouesse journalistique que constitue la Lanterne, à l'exception de l'Ordre qui brise ce qui peut apparaître comme une conspiration du silence, pour se faire le porte-parole de la presse «bienpensante».

Buies, pourtant, ne se privait point d'attaquer ses têtes de turc préférées, soit l'Ordre, soit le Nouveau-Monde, ou encore la Minerve, le Courrier du Canada et le Journal des Trois-Rivières. Il dénonce non seulement la médiocrité de la presse - et il suffit de [269]

RHAF, vol. 34, no 2, septembre 1980 
la feuilleter pour souscrire à son jugement ${ }^{1}$ - mais son étroitesse d'esprit qui maintient, selon lui, le Canada français dans un état chronique d'infantilisme politique et intellectuel: «... il n'y a rien de si outrageusement et de si stupidement fanatique que la presse canadienne-française... Nous avons des institutions libres, mais elles sont nullifiées par le fanatisme et le servilisme de la presse française. Aucune idée indépendante n'y peut se faire jour. $\aleph^{2}$.

Si Buies entend pourfendre la bêtise et l'hypocrisie, - au sens où l'entend Flaubert - il compte faire avant tout de la Lanterne «un instrument puissant de la presse, sans jamais perdre son caractère qui est essentiellement humoristique» ${ }^{3}$. La Lanterne ne devint pas «un instrument puissant de la presse», mais son humour ne fait point de doute. Buies a l'ironie mordante, manie la plume avec verve, et il est indéniable que son journal apporte du neuf dans le domaine du journalisme canadien-français. Cette nouveauté en séduisit plus d'un puisque, dans la préface du numéro deux, l'auteur annonce avec fierté un tirage de huit cents exemplaires qui «fait présager un brillant avenir» ${ }^{4}$, tandis que le numéro trois atteint le chiffre de douze cents. Cependant, n'est-ce point en partie cette nouveauté qui finit par avoir raison de l'enfant terrible du journalisme canadien? Peut-on affirmer impunément, au XIXe siècle, ou même de nos jours, que «les seules canailles sont les honnêtes gens»" et que "l'éducation cléricale est le poison des peuples» ${ }^{6}$ ?

Contrairement à ses voeux les plus chers, Buies ne trouva point les jeunes talents susceptibles d'alléger son fardeau, et il rédigea solo, ou presque, les vingt-six numéros de la Lanterne. Lecteur infatigable, il parcourait les journaux de son temps avec rage,

1 Jean Charbonneau dans Des influences françaises au Canada, II, chapitre 14: 31112-13, écrit à ce propos: «Né journaliste, il (Buies) déplorait la pénurie du journalisme et, toute sa vie, il s'insurgea contre l'esprit peu littéraire de nos quotidiens remplis, jour par jour, d'insuffisantes et prétentieuses petites chroniques étalant de vieilles rubriques, et privés de véritable critique littéraire. Buies s'est évertué à faire comprendre à ses nombreux lecteurs que la presse est supérieure, d'autant qu'elle possède de fortes qualités littéraires. Improvisés, la plupart du temps, les journalistes, nous dit Buies, ignorent leur métier parce qu'ils manquent d'une forte culture, de cette culture dont le journalisme doit faire une véritable tradition. Dans ce pays comme dans bien d'autres d'ailleurs, les journalistes de la qualité de Buies sont assez rares.»

2 La Lanterne, 118.

3 La Lanterne, 18.

4 La Lanterne, 17.

5 La Lanterne, 16.

6 La Lanterne, 133. 
y trouvant ample pâture à ses attaques les plus vitrioliques. Il est intéressant de constater que, à l'instar de Flaubert, la Bêtise faisait, si l'on peut dire, ses délices. Cette Bêtise, qu'elle prenne la forme bourgeoise en France ou cléricale au Canada, il la démasque, la traque et ne la lâche point avant de lui avoir donné le coup de grâce.

L'examen des répliques de l'Ordre aux joyeuses attaques de la Lanterne révèle à la fois la lourdeur de son style et la faiblesse de son argumentation. L'Ordre n'engage jamais le débat sur le fonds de la question religieuse au Canada, à savoir l'omnipotence du clergé, mais assène à son adversaire des vérités ex cathedra du type: «... si vous voulez voir des sottises dans les institutions véritables d'une société de plus de deux cents millions d'hommes vous êtes un sot vous-même d'oser vous attaquer à des institutions que ni les railleries, ni les sarcasmes, ni même les persécutions n'ont pu détruire» ${ }^{7}$, ou encore: "Les femmes, quoi qu'en en dise et quoi qu'on en espère, ont l'esprit et le coeur catholiques, comme par instinct. C'est l'Evangile qui a fait leur éducation et leur a donné le rang qu'elles occupent dans l'échelle sociale et au sein de la famille, et l'Évangile est catholique. $)^{8}$

Un des attraits de la Lanterne et sa «force de frappe» en quelque sorte, c'est l'esprit. L'Ordre, réflétant d'ailleurs en cela une des carences de la presse de l'époque, en est totalement dépourvu. Qu'en en juge plutôt: "A-t-on jamais vu pareille audace? Prétendre éclairer les gens avec une lanterne en plein dix-neuvième siècle! $»^{9}$ ou encore: «À propos, nous avons trouvé par hasard l'autre jour en feuilletant un dictionnaire le mot: Buie. Si vous voulez, il n'y avait pas d's; mais c'était au singulier, comme de raison. C'est un vieux mot qui désigne ni plus ni moins qu'un de ces vases vulgairement nommés: cruches. Nous n'oserions affirmer que notre confrère tire son nom de là. Ce qui nous le ferait croire pourtant, c'est que à lui seul il vaut plusieurs cruches, d'autant plus qu'il est rempli de toutes les vilenies qu'on lui a jetées. Alors, c'est une cruche d'une grande capacité. ${ }^{10}$

Les journaux bien-pensants évitent prudemment la dialectique redoutable de leur adversaire et préfèrent concentrer leurs

\footnotetext{
7 L'Ordre, 4/12/68.

8 L'Ordre, 4/12/68.

9 L'Ordre, $19 / 10 / 68$.

10 L'Ordre, 4/12/68.
} 
attaques sur «l'homme» et non sur ses arguments. Pareille tactique permet de discréditer un adversaire en se gardant bien de discuter ses thèses. Ainsi, on retrouve dans les colonnes de différents journaux les épithètes suivantes concernant Buies: bambin malapris, atrabilaire, éternel mécontent, redresseur de torts, chien hargneux, envieux, mesquin, vaniteux, menteur.

Là où Buies tente d'apporter des faits, l'Ordre et consorts n'offrent que l'insulte ou l'ironie facile. L'Evénement, par exemple, dans son numéro du 17/10/68 fait une parodie de la Lanterne, l'intitulant «Le Fanal», et Le Canadien du 21/10/68 révélant un entrefilet de la Gazette de France, repris par les journaux de New York annonçant à ses lecteurs l'existence d'une Lanterne canadienne, d'ironiser: "Les quelques lignes de cette publication, que nous trouvons dans les journaux de New York, font supposer qu'en véritable lanterne sourde, elle laisse dans l'obscurité la plus complète celui qui la tient à la main.» Quant au Nouveau-Monde du 9 décembre 1868, il déclare en conclusion de son communiqué consacré à Buies: «Monsieur Buies, mieux que personne, vous pouvez nous plaindre d'avoir peu d'esprit, car, mieux que personne, vous savez par vous-mêmes (sic) combien il est pénible d'en manquer.»

À vrai dire, tout sépare Buies de ses adversaires: intelligence, culture, expérience, (le séjour européen de Buies l'a évidemment très marqué) et, comme le dit si candidement l'Ordre: "Avec de pareilles gens, on ne discute pas: il vaut mieux les laisser dans l'ornière d'où ils ne veulent pas sortir. $\rangle^{11}$ On imagine aisément Buies faisant flèche de tout bois et lacérant ses ennemis de ses sarcasmes: "L'Ordre appelle les zouaves du pape qui écrivent de Rome des correspondants pontificaux!» Lecteurs, je vous annonce pour la prochaine Lanterne un article qui m'est envoyé par mon correspondant impérial, établi à Paris.» ${ }^{12} \mathrm{Ou}$ encore: "Ce qui m'étonne, c'est que le Canada ne soit pas peuplé aujourd'hui exclusivement de bêtes, mais là de vraies bêtes, allant à quatre pattes, car il y en a assez de ceux qui n'en ont que deux. Il faut que notre peuple ait une intelligence hors ligne pour avoir résisté depuis dix ans à des journaux comme le Nouveau-Monde, le Courrier du Canada, le Journal de Trois-Rivières, l'Union des Cantons de l'Est et la Minerve dans laquelle je lisais, l'autre jour, cette annonce: «On

11 L'Ordre, 24/12/68.

12 La Lanterne, 202-203. 
demande un jeune homme pieux dans un magasin de chaussures». Comment trouvez-vous ce rapprochement du cuir et de l'eau bénite? Quelle consolation pour les gens bien-pensants que d'enfermer leurs pieds dans les chaussures vendues par un jeune homme qui a fait ses pâques $($ sic $) .{ }^{13}$

L'accueil favorable que reçut la Lanterne dans la presse anglo-saxonne constitue pour ses adversaires la preuve irréfutable de son caractère nocif, voire diabolique. Ce qui pousse Buies à leur rétorquer: «Est-ce ma faute à moi, si vous êtes tellement bornés et tellement bigots, qu'il me faille avoir recours à une feuille anglaise pour publier ce que je crois être utile et juste... Il est temps que... les cultes ne soient pas ennemis, du moins s'ils ne sont pas semblables et qu'une intolérance odieuse, fruit de l'ignorance des uns et du bigotisme intéressé des autres, ne soit pas la règle qui domine dans nos actes... Avec ce fanatisme de la presse française, il est impossible que nous formions jamais en Canada une nation unie, et c'est vous qui maintenez cette division que le progrès de notre époque aurait dû depuis longtemps effacer. $\gg^{14}$

Buies exprime à maintes reprises son admiration pour le Canada anglais et les États-Unis, autre caractéristique qui le distingue de ses confrères, dont la majorité entretiennent encore une illusion de supériorité culturelle et spirituelle vis-à-vis des anglosaxons, illusion que Buies dénonce. En effet, les progrès de l'industrie et du commerce aux États-Unis et au Canada anglais risquent de faire de ses compatriotes des citoyens de seconde zone dans une Amérique du Nord qui entre résolument dans l'ère industrielle, alors que le Canada français s'accroche à un rêve de société agraire et théocratique, dont les privilégiés sont le clergé et une petite bourgeoisie avides de leurs privilèges.

Le combat mené par Buies revêt un caractère d'autant plus pathétique que ses adversaires ne comprennent rien à ses intentions. Les épithètes utilisées par l'Ordre pour qualifier la Lanterne: "ignoble et impudique», "pamphlet impic», "feuille immonde», indiquent bien le dialogue de sourds qui s'est engagé. L'Ordre n'est même pas de mauvaise foi: il ne comprend tout simplement pas les objectifs poursuivis par la Lanterne. Les invectives volent

13 La Lanterne, 57.

14 La Lanterne, 118. Signalons que le Witness, le Messager Franco-Américain, le Chronicle, le Globe, le Meschacebé de la Nouvelle-Orléans et le New York Mercantile, entre autres, publient des échos favorables à la Lanterne. 
de part et d'autre, la colère et la frustration s'accumulent, mais le fossé reste entier.

Bref, la presse se montrera assez indifférente à la Lanterne, et même des journaux idéologiquement proches de Buies, comme le Pays, ne lui apporteront pas le soutien qu'il méritait. Ce qui ne veut pas dire, par ailleurs, que la Lanterne a été un échec car, indéniablement, elle a introduit un élément de qualité au sein de la presse canadienne-française. Mais ce qui se dressait contre Buies était moins un mur d'hostilité qu'une apathie profonde. Peut-être son entreprise était-elle prématurée.

Toujours est-il qu'elle demeure un témoignage éloquent sur un des très rares grands journalistes de cette époque et, à ce titre comme à d'autres, elle mérite d'être appréciée à sa juste valeur. 